\title{
N8 $7-29458$
}
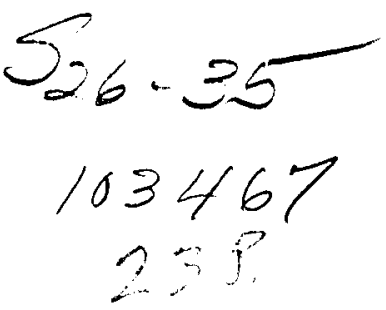

\section{PARTICLE AND FLON FIELD HOLOGRAPHY}

$\int$ A Critical Survey

\section{J. D. Trolinger}

Spectron Development Laboratories, Inc. 3303 Harbor Blvd., Suite G-3

Costa Mesa, California 92626

\section{Abstract}

A brief background is provided for the fields of particle and flow visualization holography. A summary of methods currently in use is given, followed by a discussion of more recent and unique applications. The problem of data reduction is discussed. A state of the art summary is then provided with a prognosis of the future of the field. Particle and flow visualization holography are characterized as powerful tools currently in wide use and with significant untapped potential.

\section{Introduction}

Strictly speaking, the first application of holography to the study of particles was by Dennis Gabor himself who invented holography in 1947. 1 He produced holograms of a variety of microscopic samples using partially coherent light since lasers were not available for another fifteen years. His application intended to exploit the magnification properties of holograms and not the three dimensional imaging properties. Nearly another twenty years passed before holography left the research laboratory to actually be applied in a field environment. The first such work appears to be that of Thompson and coworkers ${ }^{2}$ who used the three dimensional properties of holograms to characterize fog droplets.

The first significant work with flow visualization holography appears to be that of Brooks, Heflinger, and Wuerker who used holographic interferometry to determine the flow fleld around projectiles in flight. ${ }^{3}$ During the past twenty years virtually every test facility in the world with a need for flow diagnostics has incorporated holography to some extent, with some measure of success. Hundreds of publications have reported new innovations, techniques, refinements, and hardware. Many of these amounted to laboratory feasibility demonstrations of a new variation on recording or data extraction and never made it outside the laboratory. One of the first major test facilities to incorporate holography into an operational wind tunnel was the Arnold Engineering Development Center in Tennessee. ${ }^{4}$ Research facilities of NASA, Army, Air Force, Navy, and aerospace companies began using br at least exploring the possibility of using the techniques during the early 1970's. European and Asian countries also began incorporating this technology into such facilities. 
Unfortunately, the development of practical data handling techniques lagged far behind what was required to compete with conventional photographic techniques. 5 Holography systems required expert operators and they required more of almost everything when compared with the highly refined science of photographic recording. The result was that the use of holography as a routine tool actually seemed to decline in the major test facilities by the late 1970's. Flow visualization holography has not replaced more conventional methods, but it has become more of a compliment. When a very distinct set of conditions or requirements obtain, flow visualization holography is often the best, or least expensive, or quite often the only way to obtain a desired set of data. It is usually, but not always, the more difficult method since it usually involves less refined, more complicated hardware.

Particle holography has satisfied a need which is so basic to the study of particle fields that its use has continued to grow steadily since the first application. 6 Even though the data handling problem has not been totally solved here either, the number of applications requiring the features of holography has continued to fuel its development and use. With present technology, holography is the only recording method by which one may record an accurate three-dimensional image of a dynamic event. This is particularly useful, for example, in the microscopic examination of combustion and explosive events, droplet breakup and formation mechanisms, and velocity measurement of droplet fields.

Beyond this brief historical survey the intent of this survey is to examine the current status of particle and flow field holography, including a summary of the present state-of-the-art, a summary of the applications and where they are being made, and a prognosis for the fucure. Particle and flow field holography are commonly treated together since many of the applications involve both technologies. However, the characteristic requirements for holocameras and data reduction for the two techniques are so different that they must be described individually.

\section{A summary of methods}

Particle Field Holography

There are two types of recording procedures for recording holograms of particle fields: in-line holography and off-axis holography. With in-line holography a simple wavefront ( $p$ lane or spherical) is passed through the particle field, which must be about 80 percent transparent for this to be successful. Some of the light is scattered (the object wave) by the particles and some of the light passes through unscattered (the referenced wave). These two waves interfere, creating a diffraction pattern which when recorded is the hologram. With of $f$-axis holography, light which has scattered from the particle field is mixed with a mutually coherent wave which has taken a second path around the particle field (the reference wave). 
A second level of classification for both types of holography comes with the use of lenses or mirrors to image the particle field and producing a hologram of this image (appropriately termed image holography). A wide range of imaging systems may be chosen depending on the special needs of a given experiment. Imaging is commonly done to impart a convenient size, location, or magnification to the field of interest, or to relax the required recording capabilities of the hologram.

Off-axis holography is much more versatile with respect to type of object illumination. off-axis holograms can be categorized according to whether the object illumination is a simple wavefront (plane or spherical), a diffused wavefront, or a structured wavefront. The most common type of structured wavefront in current use is the so-called "sheet of light" produced with a cylindrical lens and a small diameter collimated beam of light. Figure 1 is a categorization of the possible types of recordings that have unique characteristics. Clearly, a large number of combinations are possible. The holocamera itself must be engineered to accomplish the following tasks:

1. Properly illuminate the field of interest.

2. Place the interest field or its image in a suitable place relative to the hologram.

3. Achieve an acceptable F-number for the desired resolution.

4. Deal with environmental factors.

5. Meet coherence requirements.

6. House the film or plate transport, laser, and optics

7. Produce a hologram of acceptable quality.

The reconstruction system must be compatible with the holocamera and must be designed to produce a reconstructed image which is sufficlently bright, noise free, aberration free, the right size, and in the right location for analysis. An extremely important part of this system must cast the data into a useful form. The images may be recorded as photographs, or analyzed directly by an image analyzer.

The question that is often asked is "How many photographs are in a hologram?" This can actually be computed to some degree of accuracy. The resolution limit in an imaging system is given by

$$
R \approx \frac{5}{D} \lambda
$$

where $S$ is the object distance, $D$ is the diameter of the imaging system, and $\lambda$ is the wavelength of 1ight. (See Figure 2).

The depth of fleld in such an image is given by

$$
\delta *\left(\frac{\mathrm{S}}{\mathrm{D}}\right)^{2} \lambda \approx \frac{\mathrm{R}^{2}}{\lambda}
$$




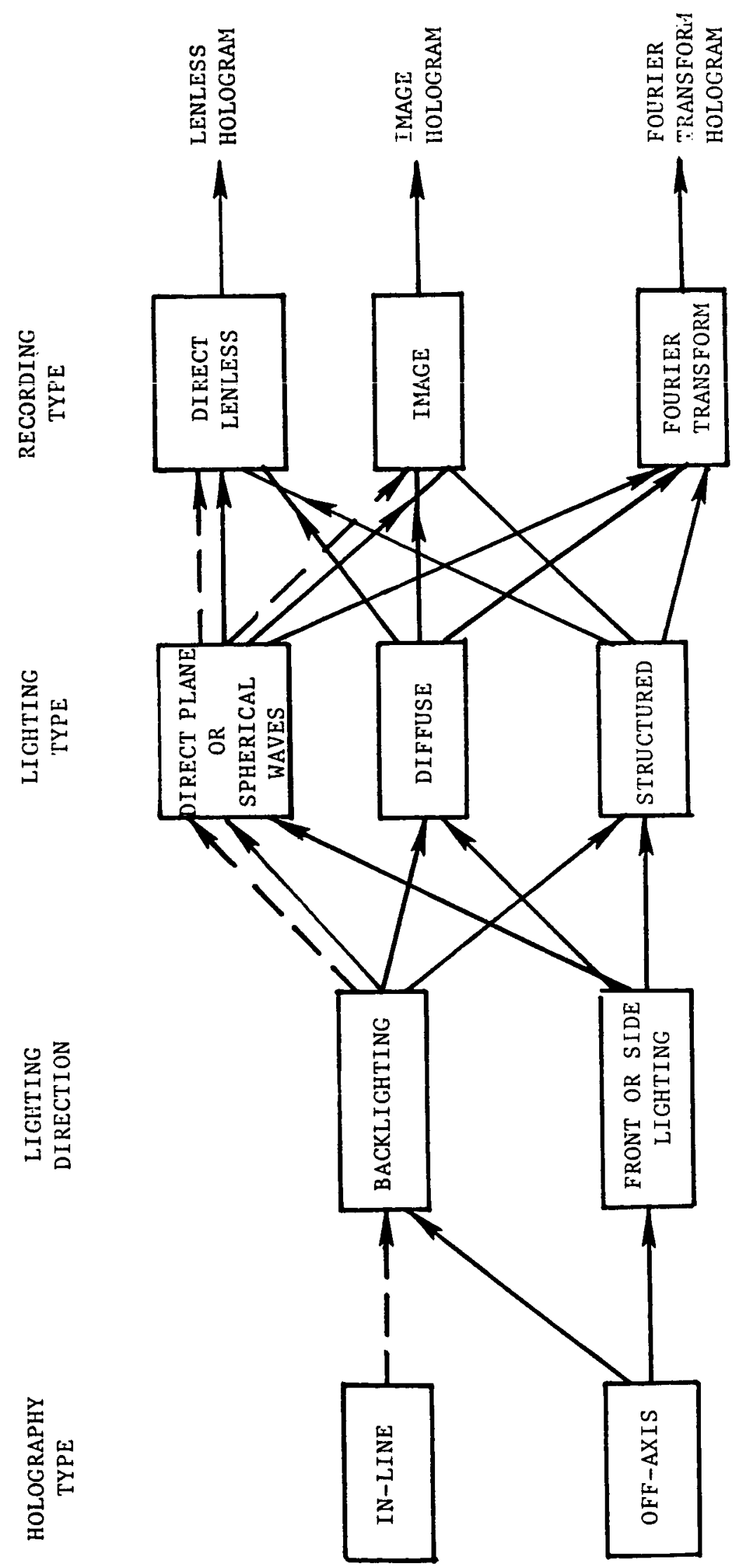

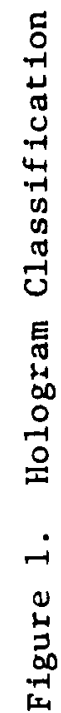




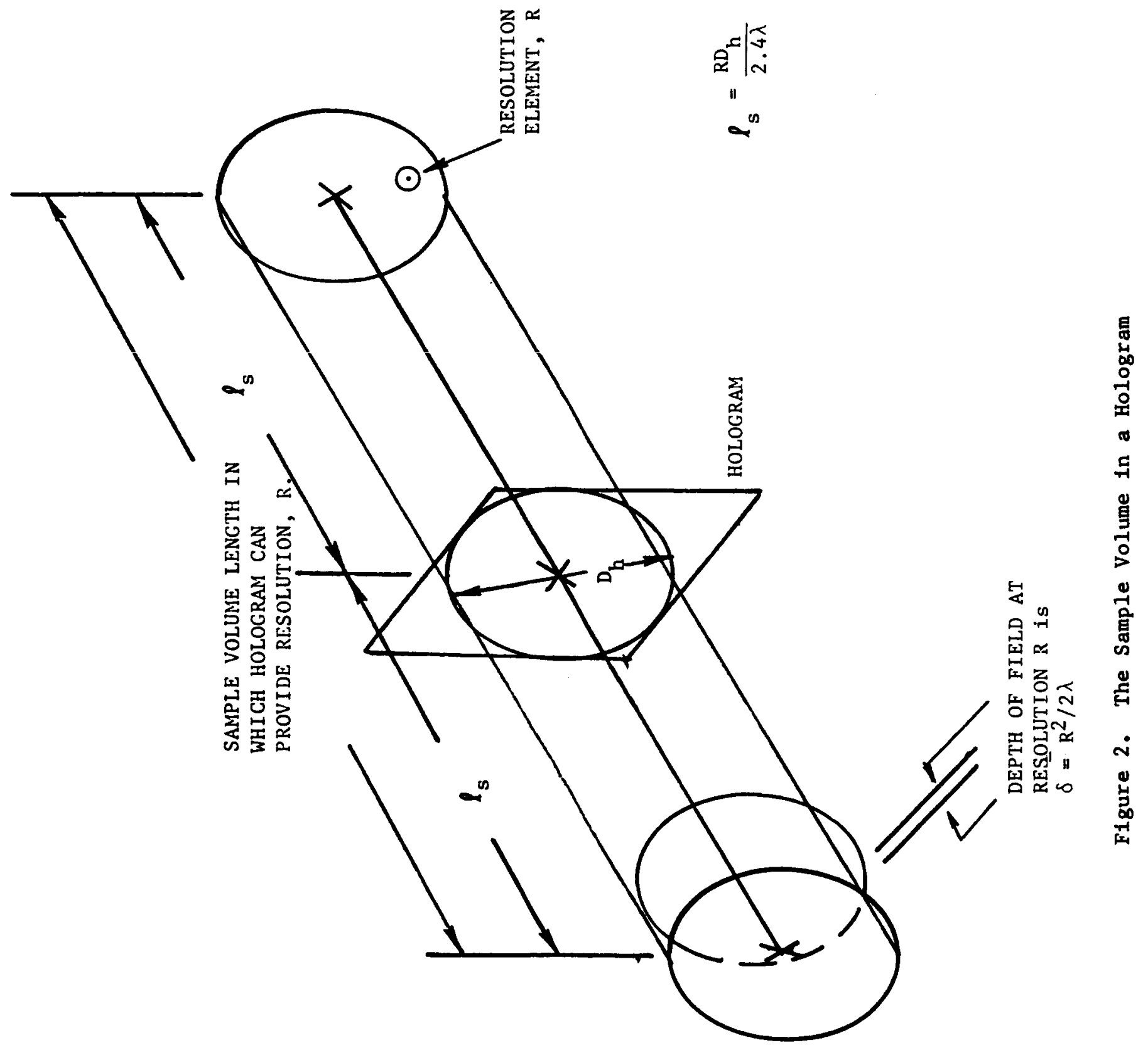


A perfect hologram of diameter, $D_{h}$, can resolve an image of size, $R$, if it lies within a distance $R D / \lambda$. The ratio of the two sample volumes is therefore equal to the number of distinctively different photographs which can be produced from the hologram and is approximately

$$
\mathrm{n} \approx-\frac{2 \mathrm{D}}{\mathrm{R}}
$$

When one considers a study of particles of 10 micrometers in diameter in a typical $10 \mathrm{~cm}$. diameter hologram, this number comes to a staggering 20,000 photographs, not counting the photographs which could be taken outside of the sample volume at lower resolution. This provides a clue to the difficulty in data reduction.

Double pulsed holography has been used extensively to study the dynamics of particle flelds. ${ }^{7}$ Figure 3 illustrates such a hologram of the particle field of a spray nozzle. The central figure is a global view of the entire field while the insets illustrate high resolution focusing at different depths in the field." Two superimposed threedimensional fields present an extremely complicated image. A variety of ingenious techniques have been devised to overcome the complexity. By giving different exposures to the two recordings one can tell which image belongs to which exposure. Still more elegant methods record the two exposures with two different reference waves or even two different wavelengths. Then during reconstruction the two 1 mages can be viewed independently. The so-called "flicker method" 8 shifts back and forth between the two 1 mages allowing one to observe the motion. Still another alternative is to reconstruct the two images with reference waves which have been phase- shifted by 180 degrees so that the images will subtract, eliminating the constant noise which would otherwise appear in both recordings and which would otherwise add goherently with the signal, severely reducing the signal-to-noise ratio. 9

Holocameras can be equipped with any laser which meets coherence requirements; however, except for rather slow moving distributions, pulsed lasers are required. Most commonly, frequency doubled YAG or ruby lasers are used. In recent years, YAG lasers have become more popular because of their ability to operate at higher rep rates. Typical pulse times for YAG lasers are tens of nanoseconds and rep rates are typically tens per second, as compared to ruby lasers with typical rep rates of a few per minute.

Flow Visualization Holography

In flow visualization, the object is usually a phase object and therefore off-axis holography is almost always required. The wavefront which has passed through the flow field and has been modulated by the field can be recorded in its entirety for later analysis. Later analysis may be accomplished by any of the conventional methods of wavefront analysis. Such methods include shadowgraph, Schlieren, deflectometry, 


\section{- por OUALTS}

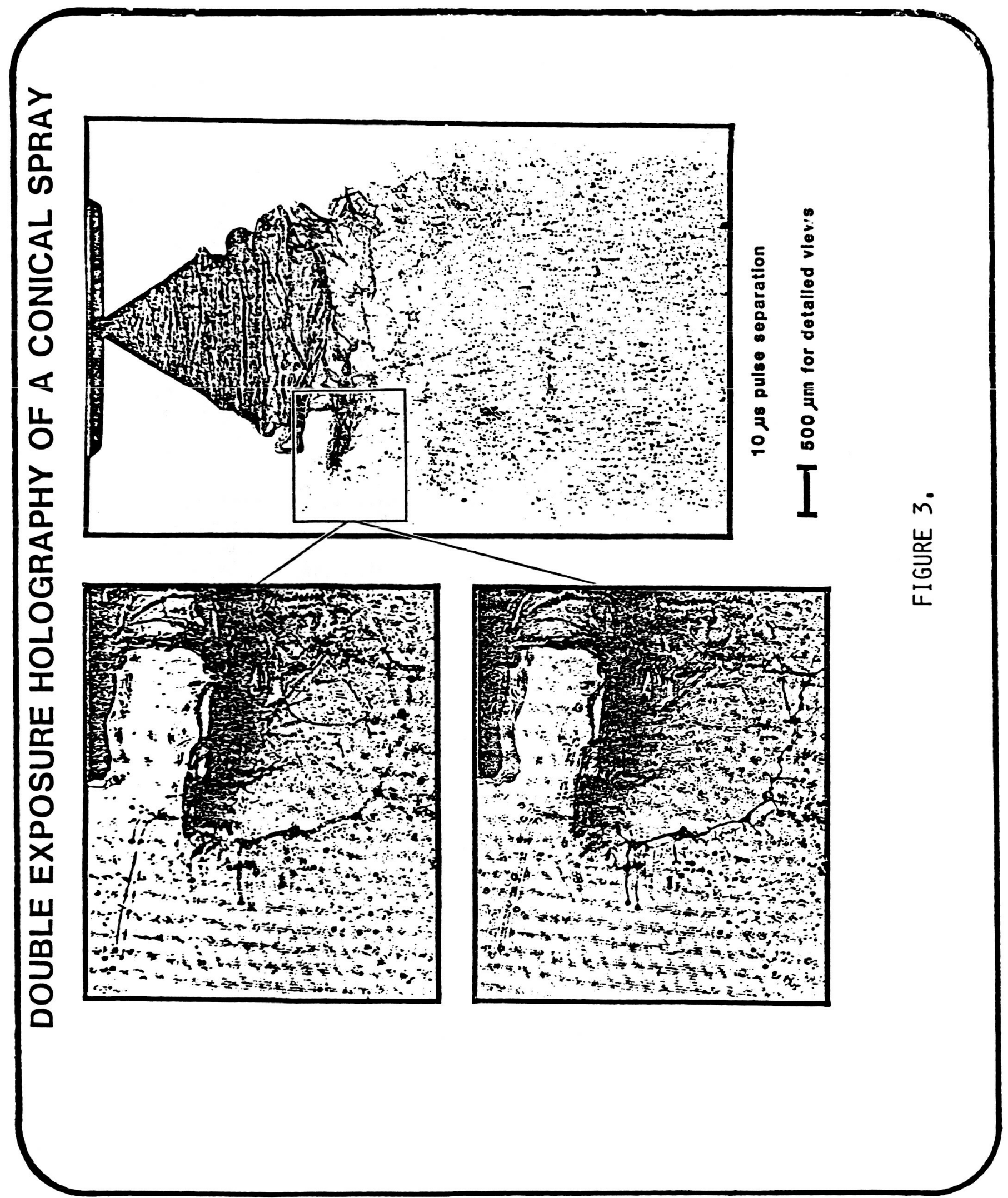


Hartmann, moire, or interferometry. A significant fact is that one does not have to select which of these will be used until later. Any, or all, of these can be applied to the reconstructed wavefront.

An extremely important technique is to store a wavefront which has been modulated by the optical system only, including the windows in the test facility for later comparison with a wavefront which is also modulated by the flow fleld. In this way, the effect of the optics can be subtracted from the measured wavefront exactly, allowing the use of low cost optics. Figure 4 briefly summarizes holographic interferometry options for flow diagnostics. Each of these methods has some unique characteristic which makes it ideally suitable for some specialized application.

Figure 5 illustrates the typical flow visualization holocamera, commonly bult into a conventional flow visualization system. The system is broken into elements with the possible choices of components listed to illustrate the characteristic state-of-the-art system.

\section{Current applications}

This section is intended to provide a summary of current applications and where they are being conducted. At the present time, the use of holography is so wide spread that every application cannot possible be covered here. So this must be considered a highlighting of applications and apologies are offered to those who have been onitted.

Since some applications involve simultaneous particle and flow diagnostics, the discussion here is not exactly divisible into the two areas. Therefore, some intermixing of the discussions seemed inevitable.

Flow Diagnostic Applications

A significant feature of many modern day applications, which has changed from the past, is the number of holograms and recording rate. Early applications of holography commonly amounted to the recording of a few holograms during a day or in extreme cases, a few hundred. In today's experimental applications it is not uncommon to record thousands or even tens of thousands of holograms. This has placed even greater demands on data reduction. Table 1 provides a summary of applications of particle and flow visualization holography, with emphasis primarily on U.S. Government laboratortes and universities. Some of these will be discussed in more detail here. An exciting NASA application by Marshall Flight Center is scheduled in the Space Lab $3^{10}$ space shuttle mission for May, 1985. This will be the first application of holography in space. The system's somewhat unique design was required by the nature and constraints of the space shuttle laboratory. The application is to observe the growth of a crystal in zero gravity and to measure density gradients in the solution surrounding the crystal. Two holocameras and a Schlieren system are combined into a single optical system. 


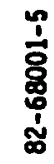

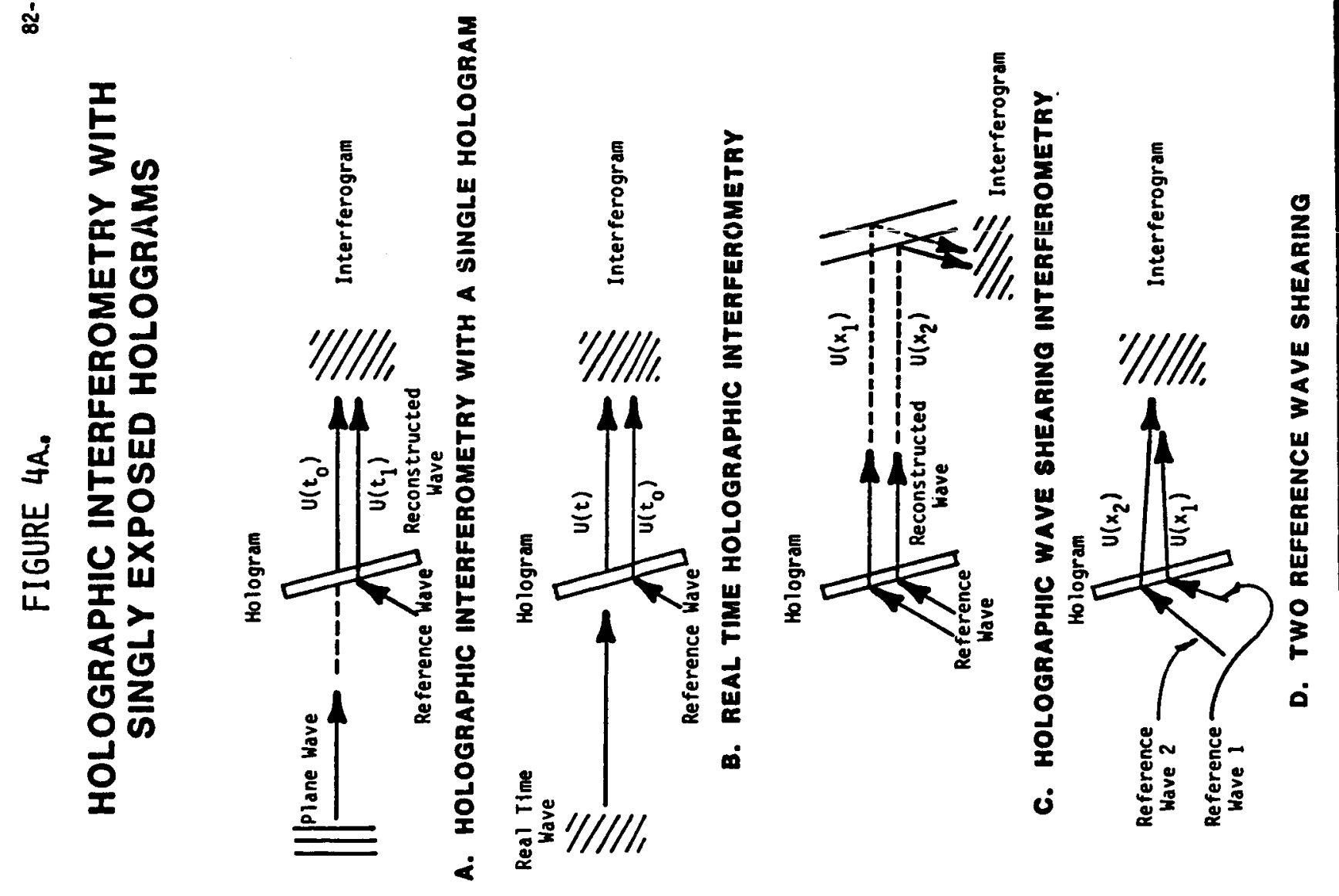




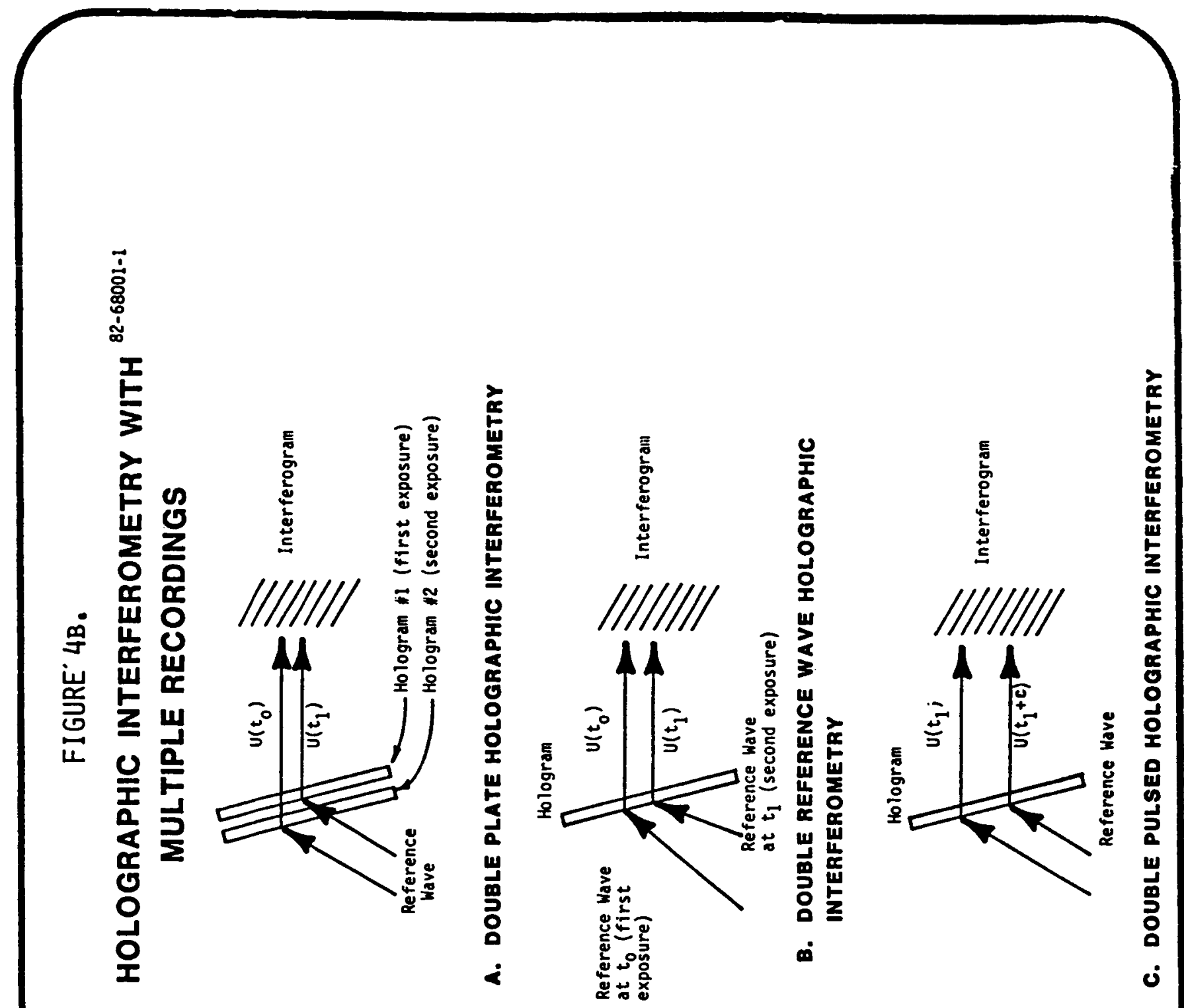


85-2421-02

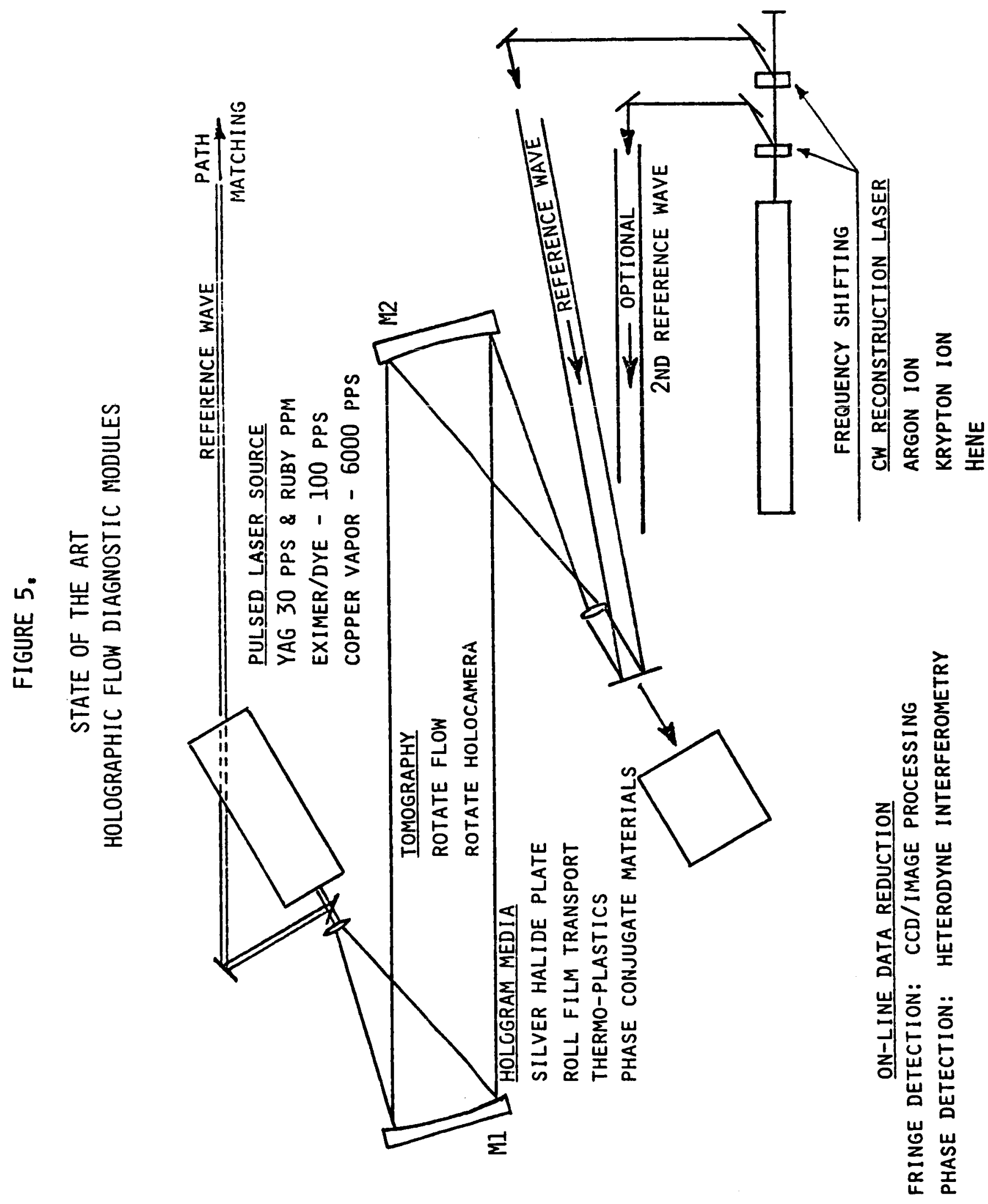


TABLE 1 - Application of Particle and Flow Visualization Holography in U.S. Government Laboratories

1. Particle Sizing

A. Icing Facilities

B. Spray Nozzles

C. Combustion

D. Particle Break-up

F. Explosives

F. Impact Facilities

G. Crystal Growth

H. Meteorology

I. Pollution

J. Multiphase Flow

K. Fiberization

L. Flow Seeding

\section{Flow Diagnostics}

A. Wind Tunnels

B. Gun Ranges

C. Shock Tubes

D. Combustion

E. Plasumas

F. Tomography

G. Movies

H. Water Tunnels

I. Test Chambers

J. Diffusion

K. Rocket Exhausts

Users

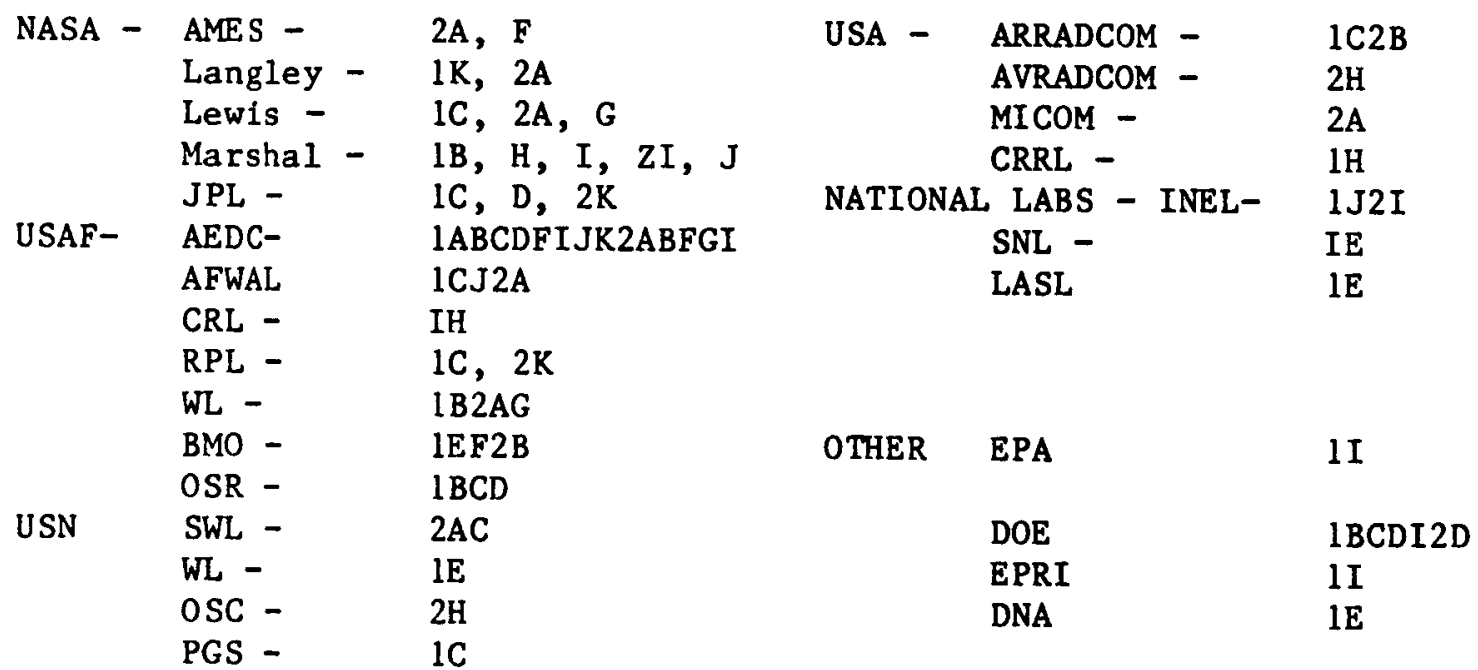


For one holocamera, the crystal is side lighted with a choice of direct or diffuse illumination. This will provide a view of the crystaline surface and facets in three dimensions with a resolution of about 30 micrometers.

The second holocamera, which is for flow diagnostics, backlights the crystal and surrounding fluid with direct light. The reconstructed wavefront will be diagnosed for density gradients near the crystal surface. The holocameras operate with a single helium neon laser and holograms are recorded on Kodak SO $103 \mathrm{film}$ which is transported by a vacuum back which holds the film flat during recording.

Other significant developments within NASA include holographic movie capability at high framing rates 11 at the Lewis Research Center. This system employs a frequency doubled YAG laser pulsing at framing rates up to twenty per second. Future plans include significant increase in this recording rate. The primary applications to date has been in the study of flow fields over turbine blades and in combustion.

Another unique, but somewhat Indirect application of holography at the Lewis Center, Involves the use of a holographic optical element in a Laser Doppler Velocimeter to provide the required beam splitting operation while at the same time performing aberration correction for observing flow fields inside of cylindrical chambers. 12

The NASA Ames Research Center has evolved wind tunnel holography for nearly fifteen years 13,14 , and currently has operational flow diagnostic holocameras in several facilities. The current systems employ frequency doubled YAG lasers with various types of holographic interferometry. One of these systems is a permanent installation integrated into a conventional sixty $\mathrm{cm}$. diameter Schlieren system. The Ames applications have been especially significant since they have been complimented with extensive use of other diagnostic instruments such as laser velocimeters and aerodynamic probes. The NASA Langley Center has likewise applied holographic interfer-
ometery for flow diagnostics in a variety of wind tunnel applications.

The U.S. Army applications, almost as extensive as NASA, include a number of extremely important efforts. A highly advanced holography system is operated by the U.S. Army Aeromechanics Laboratory at Moffet Field, California. This system is capable of performing holographic interferometry of a $70 \mathrm{~cm}$. diameter flow field around a helicopter rotor tip. This system is so configured to provide a "look" through the flow field at any angle of view and has been used routinely for tomography of the field of interest, producing three dimensional density distributions. This appears to be the largest operational holographic tomography system in use today. It is producing three dimensional density data of the flow around helicopter blade tips, data which has never been available before. 
Army applications of flow diagnostic holography have also been made at Picatinny Arsenal, Redstone Arsenal, and Aberdeen Proving Ground, and the U.S. Army Research Office has played an important role in supporting research and development of the techniques.

The U.S. Air Force has been instrumental in the development and application of flow diagnostic holography, especlally at the major wind tunnel test centers, Arnold Engineering Development Center ${ }^{17}$, and Wright Aeronautical Laboratory ${ }^{18}$. The Air Force office of Scientific Research has been instrumental in advancing the technology 19 . A unique application by the Air Force Weapons laboratory resulted in the development of an airborne holocamera for the diagnostics of flows over cavities and turrets. 20 The ultimate effect under study is the aberration of optical wavefronts by aerodynamic flow fields, a relatively new area of study known as aero-optics. Whereas the usual application is the diagnostics of the flow by studying the phase shifting of an optical beam, aerooptics closes the loop by determining the effect of the flow field on propagation and then examining the ways to achieve some optimum by modifying either the wavefront or the flow fleld. This particular holocamera system is unique in that it has been employed in an aircraft and is backed up by an automated data reduction system capable of reducing the thousands of holograms produced ${ }^{21}$. The Alr Force Wright Aeronautical Laboratory is currently developing a combined flow diagnostic holocamera and laser velocimeter for application in a transonic wind tunnel.

The U.S. Navy has developed and applied flow diagnostic holography at the Surface Weapons Center and the Oceans Systems Center.

The major aerospace aircraft and aircraft engine companies continue to use holographic interferometry for flow diagnostics. A somewhat unique system employed by Rolls Royce Limited produces large area holograms of the flow between fan blades in jet engines ${ }^{22}$.

National Laboratories have used holography and continue its use in a variety of instances. For example, the Idaho National Engineering Laboratory has applied holography in two phase flow and in electric arc diagnostics ${ }^{23}$, Sandia and Livermore National Laboratories in combustion ${ }^{24}$, Los Alamos National Laboratgry in nuclear fusion research, Bureau of Standards in combustion. 25

Other Particle Sizlng Applications

Applications in particle sizing are even more widespread than in flow diagnostics, although the areas do overlap somewhat. Almost all of the laboratories mentioned above have also applied particle sizing holography. The basic capability is the high resolution diagnostics of a three-dimensional particle field providing size, shape, number, and velocity of particles.

Areas of application include: 
1. Nozzle development and diagnostics

2. Fuel characterization

3. Combustion and explosions

4. Meteorology

5. Pollution and contamination

6. Multiphase flow

7. Fiber generation.

8. Icing facilities

9. Particle Break-up

10. Impact

i1. Flow seeding

A few of the more recent or unusual works will be mentioned here. Holography has become more field oriented and airborne applications are becoming more common. 10,26,27 Meteorological applications allow a microscopic look at ice crystals in clouds distributed over a large volume. Particle break-up, a phenomenon which escapes photography, is simple with holography, even at high speeds as observed from aircraft or in test chambers. 19

Observing detailed particle optical properties with holography is a relatively new technology. 28

Field holocameras which are rugged, lightweight and portable ${ }^{29}$ are not yet in widespread use, but the necessary technology now exists.

Considerable attention has focused on reşolution and signal-tonoise improvements in particle holography. $30,31,32$ Noise is still a critical factor limiting useful resolution to considerably worse than direct two dimensional microscopy.

Three dimensional velocity fields have been determined through holography from many years. However, new techniques continue to evolve. Murakami, et al, for example, developed a two-color multiple exposure holography system whigh produces two separate time spaced holograms of the same volume. 33

In general, the three dimensional, high resolution capability which is made possible by holography, should continue to make this technique unmatched by any other for particle field diagnostics. Automatic data reduction will also provide new impetus to this field.

Automated data reduction technology

One of the most severe obstructions to the widespread use of holography has been data extraction and reduction of the vast amount of data which is stored in a typical hologram. Studies have too of ten ended 
with production of holograms, a few photographs, and little of painstaking effort necessary to go further. In recent years great strides have been made at removing the obstruction. Available computer power, new codes, and detector arrays have played a key role.

The problem has two major facets, dealing with vast quantities of data, and dealing with optical noise. Holographic images are characterized by speckle, interference, and diffraction noise that too often has the appearance of signal. Out of focus images also become noise when in focus images are examined and all optical noise adds coherently, amplifying the problem. The numbers of dimensions in the general problem includes three for location, two or three for morphology, two for velocity, four for interferometry, and two more for tomography. For a volume search at high resolution this quickly adds up to exceed the storage capacity, even of large computers. Therefore, data compression and rejection schemes are required. The general problem of automation is too complex and the most successful schemes require considerable operator interaction. Figure 6 illustrates the typical data flow and Table 2 explains the various functions required.

TABLE 2. DATA REDUCTION FUNCTIONS

- Image Analysis -

(1) Number of each fringe

(2) Locate the center of each fringe

(3) Produce XY coordinates for each fringe

- Image Processing -

(1) Connect broken fringes

(2) Filter out optical noise

(3) Subtract off uneven background

(4) Allow input to correct ambiguities

- Data Analysis

(1) Determines deviation of fringe from a reference line

(2) Produces fringe deviation (phase) map $\phi$ $(\mathrm{x}, \mathrm{y})$

- Data Interpretation - (1) Solves an integra1 - Radon transform General

axisymmetric

- Abel integral -

dimensional

- Linear equation - two

(2) Derives refractive index $N(X, Y, Z)$

(3) Converts $\mathrm{N}(\mathrm{X}, \mathrm{Y}, \mathrm{Z})$ to desired parameter, e.g. concentration 
(4) Determines coefficients of series representations of $\phi(X, Y)$

(5) Provides graphic plots of parameters

A number of laboratories now have operational automated data reduction facilities that have successfully addressed the requirements for holography, and this could be the basis for a new surge in its application. 5

The current state-of-the-art

The current state-of-the-art frontiers are summarized in Table 3 including some of the associated technolugy or hardware that is 1ikely to be involved in the advancement of the state-of-the-art. The major advancement in the state-of-the-art in recent years has been assoctated

TABLE 3. STATE-OF-THE-ART FRONTIERS AND ASSOCIATED TECHNOLOGY

Parameter Technology or Hardware

$\begin{array}{ll}\text { Recording Times } & \text { Mode Locking, Pulse Slicing } \\ \text { Recording Rates } & \text { YAG, Excimer, Metal Vapor Lasers } \\ \text { Sensitivity } & \text { Heterodyne Interferometry } \\ \text { Data Extraction } & \text { Thermoplastics, Phase Conjugate Materials } \\ \text { Storage } & \text { Nonlinear Materials } \\ \text { Data Reduction } & \text { Detector Arrays, Software, Computer Power } \\ \text { Resolution } & \text { Recording Technique, New Materials }\end{array}$



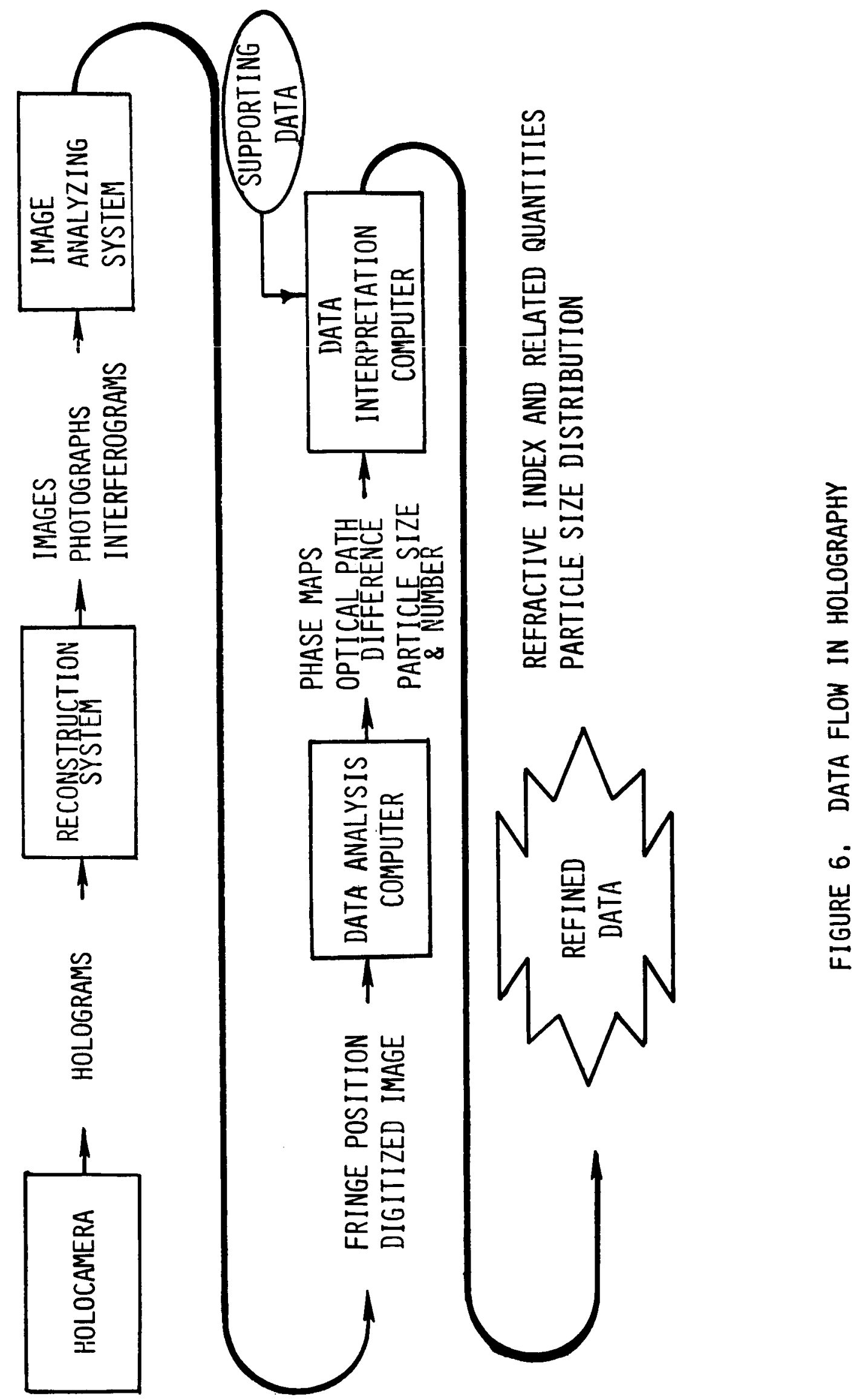
with automated data reduction. The ability to produce holograms of particle and flow fields which contain useful data has for years exceeded the ability to conveniently extract the data. This condition has been relaxed somewhat by the advent of advanced image analyzers, computers, and software. However, the process, unfortunately is not low in cost and its availability is still extremely limited.

Pulsed laser holography is largely performed using Q-switched ruby, or frequency doubled YAG lasers with pulse times less than 20 nanoseconds. Some work has been done with dye lasers. Recording times can now be reduced by over an order of magnitude by mode locking and/or pulse slicing. This is an area where the state-of-the-art has remained unchanged for many years.

Recording rates in today's holocamera are laser limited. Where YAG lasers are used, power requirements have 1 ifited recording rates to about 30 frames per second. This limit applies only to continuous recording. A laser can be multi Q-switched at megahertz rates for a few pulses. Commercially available lasers can deliver three or four pulses with microsecond separation.

Real time or near real time recording media such as thermoplastic and phase conjugate materials have provided opportunities to use holography in some innovative ways. Commercially available thermoplastic recording devices are now in routine use. On-line electronic hologram development makes possible on-line holographic diagnostics. Currently available hardware is nearly adequate to make possible a flow or particle diagnostic holocamera which can produce finished data on line. However, such a system is still yet to be built.

Hologram resolution limits have almost reached diffraction limited in laboratory uses. In field application, this is significantly worse and a typical achievable resolution is greater than 2 micrometers for particles.

A prognosis of the future - Ideas beyond the frontier

Flow visualization holography will continue to find application largely where "nothing else works" until automated data reduction technology finds its way into more routine use. This should happen as computers and software continue to become more powerful.

The field of tomography will in the near future experience a new surge in solving flow diagnostic problems which have evaded solution for years and which are sufficiently important to justify the still tedious, though now practical, procedures.

Heterodyne holographic interferometry is an extremely high potential area which still remains virtually untapped. By combining the features of holography with the improved sensitivity made possible by heterodyning, a new, powerful diagnostic method will become available. In addition to sensitivity improvement this method offers a key link 
toward automated data reduction. At present, the problem with sufficient importance has not surfaced to provide someone incentive to develop this technology. It surely will eventuallty.

Four wave mixing has found an application in aero-optics, that of aberration correction of propagating beams. This area is so young there must be other applications in flow diagnostics.

Real time holography materials or fast processing thermoplastics are making on-line holography more practical. Within a few years, holography diagnostics will be done on-line with a system that records, processes electronically, and produces refined data in near real time.

\section{Conclusion}

The field of particle and flow visualization holography is alive and well. After an explosive beginning which fizzled, we more realistically accepted less than a miracle panacea and realized that applying holography usually meant more than just showing a reconstructed threedimensional image. Indeed, the most useful holograms in this field are not optimum for human viewing at all, but are optimized for machine viewing. There have been few major breakthroughs in the past ten years. Growth and improvement have evolved more through refinement and application of improving hardware. These, combined with significant untapped potential, should provide a bright future for serious holographers. The current extent of applications and cost and complexity of hardware are likely to constrain this to be a specialist's technology for some time. 


\section{References}

1. Gabor, D., "A New Microscope Principle", Nature 161 777-778 (1948).

2. Thompson, B. J., J. Ward, W. Zinky, "Application of Hologram Techniques for Particle Size Analysis", J. Opt. Soc. Am. 55 1566, (1965).

3. Brooke, R. E., L. O. Heflinger, and R. F. Wuerker, "Interferometry with a Holographically Reconstructed Comparison Beam", App1. Phys. Letters, 7, 248, (1965).

4. O'Hare, J. E. and J. D. Trolinger, "Holographic Color Schlieren", App. Opt. 8, 204, (1969).

5. May/June issue of "Optical Engineering" will feature Automated Reduction of Image and Hologram Analysis.

6. Trolinger, J. D., M. Azzazy, D. Modarress, and J. E. Craig, "Laser Diagnostic Methods - A Summary in Lasers in Fluid Mechanics and Plasmadynamics edited by C. Wang, AIAA Publication (1983).

7. Trolinger, J. D., and M. Heap, "Coal Particle Combustion Studied by Holography", App. Optics 18, 1757, (1979).

8. Briones, R. and Wuerker R., "Holography of Solld Proplellent Combustion", SPIE Volume 125, Advances in Laser Technology for the Atmospheric Sclences, (1977).

9. Trolinger, J. D., "Application of Generalized Phase Reconstruction to Flow Visualiation Holography", App. Opt. 18, (1979).

10. Owen, R. B., "Interferometry and Holography In a Low Gravity Environment", App. Opt. 8, 1349, (1982).

11. Decker, A. J., Measurement of Pluid Propert1es Using Rapid Double Exposure and Time Average Holographic Interferometry", AIAA 84 1461 (1984).

12. Schock, H., S. Case, and L. Konicek, "Window Aberration Correction in Laser Velocimetry Using Multifaceted Holographic Optical Elements", App. Opt. 23, 752, (1984).

13. Craig, J. E., G. Lee, and W. Bachalo, "Nd/YAG Holographic Interferometer for Aerodynamic Research", SPIE Proceedings 353, (1982).

14. Perry, R. L., and G. Lee, "Holographic Interferometry Applied to Symmetrical Aerodynamic Models in a Wind Tunnel", SPIE 523 (1985). 
15. Burner, A., "A Holographic Interferometer System for Measuring Density Profiles in High -elocity lows", 73, ICIASF Record, 140, (1973).

16. Kittleson, J., "A Holographic Interferometry Technique for Measuring Transonic Flow Near a Rotor Blade", NASA T.M. 84405, (198).

17. Trolinger, J. D. "Flow Visualization Holography", Optical Engineering 14, 470 (1975).

18. Havener, G., "The Application of Holographic Interferometry to the Measurement of Transaction in Supersonic, Axisymmetric Boundary Layers", AIAA Journa1, June (1984).

19. Craig, J. E., "Conventional and Liquid Metal Droplet Breakup in Aerodynamic Nozzle Contractions", AIAA-84-0201, 22nd Aerospace Sciences Meeting (1984).

20. Craig, J. E., J. D. Trolinger, and W. C. Rose, "Propagation Diagnostic Technique for Turbulent Transonic Flow", AIAA-84-0104, 22nd Aerospace Sciences Meeting (1984).

21. Wilkens, M., "Automatic Fringe Reduction Facility", To be published, Optical Engineering, May (1985).

22. Bryanston-Cross, P.J., "Three Dimensional Flow Visualization", In Proceedings of Symposium Measurement Techniques in Transonic Flows and Cascades (1982)

23. Key, J., "Development of Holographic Welding Arc Analysis" Proceedings of the Laser Institute of America 23rd Congress on Laser Applications, Nov. (1983).

24. Sheffield, S., Hess C., Trolinger J., "Holographic Studies of the Vapor Exlosion of Vaporizing Water in Fuel Emulsion Droplets" Proceedings of the Second International Colloquiem on Drops and Bubbles JPL Publication 82-7, Nov. (1981).

25. Kashiwaga, T., "A Study of the Radiative Ignition Mechanisms of a Liquid Fuel Using High Speed Holographic Interferometry", 19th International Symposium on Combustion/Combustion Institute, 1511, (1982).

26. Trolinger, J. D., "Airborne Holography Techniques for Particle Field Analysis", Ann. N.Y. Acad. Sc1. 267, 448 (1976).

27. Conway, B., S. Canghey, A. Bentley, J. Turton, "Sound Based and Alrborne Holography of Ice and Water Clouds", Atm. Envir. 16, 1193 (1982) 
28. Pikyl, L., C. Vest, "Holographic Imaging of Semi-transparent Droplets", App. Opt. 21, 2541, (1982).

29. Brendon, B., "Miniature Multiple Pluse Q-Switched Ruby Laser Holocamera for Aerosol Analysis", Opt. Engr. 20, (1981).

30. Briones, R., "Particle Holography at Extended distances and Micron Resolutions", Proceedings of SPIE, Recent Advances in Holography, Feb. 1980, 112 (1980).

31. Thompson, B., and P. Dunn, "Advances in Far Field Holography Theory and Applications", Proceedings Feb. 1980 SPIE Symposium Recent Advances in Holography (198).

32. Trolinger, J. D., "Analysis of Holographic Diagnostics Systems", Opt. Engr. 19, 722 (1980).

33. Murakami, T., "HoIographic Observation of Fine Particles by Two Laser Pulses of Different Wave Length", Jap. Soc. Mat. Sci. 27 (1978) 